This article is published with open access at Springerlink.com

\title{
On the local closure of clones on countable sets
}

\section{ERHARD Aichinger}

\begin{abstract}
We consider clones on countable sets. If such a clone has quasigroup operations, is locally closed and countable, then there is a function $f: \mathbb{N} \rightarrow \mathbb{N}$ such that the $n$-ary part of $C$ is equal to the $n$-ary part of $\operatorname{Pol} \operatorname{Inv}^{[f(n)]} C$, where $\operatorname{Inv}^{[f(n)]} C$ denotes the set of $f(n)$-ary invariant relations of $C$.
\end{abstract}

\section{Results}

We investigate clones on infinite sets $[10,11,5]$. For a clone $C$ on $A$, its local closure $\bar{C}$ consists of all those finitary operations on $A$ that can be interpolated at each finite subset of their domain by a function in $C$, and we have $\bar{C}=$ Pol $\operatorname{Inv} C$. Here, as in [10], Inv $C$ denotes the set of those finitary relations on $A$ that are preserved by all functions in $C$, and for a set $R$ of relations on $A, \operatorname{Pol} R$ denotes the set of those finitary operations on $A$ that preserve all relations in $R$. A clone is called locally closed if it is equal to its local closure. $C$ is called a clone with quasigroup operations if there are three binary operations $\cdot, \backslash, / \in C$ such that $\langle A, \cdot, \backslash, /\rangle$ is a quasigroup [3, p. 24]. Theorem 1.1 states that a clone with quasigroup operations on a countable set is either locally closed, or its local closure Pol Inv $C$ is uncountable.

Theorem 1.1. Let $A$ be a set with $|A|=\aleph_{0}$, and let $C$ be a clone with quasigroup operations on $A$. If $|\operatorname{Pol} \operatorname{Inv} C| \leq \aleph_{0}$, then $C=\operatorname{Pol} \operatorname{Inv} C$.

This theorem does not hold for clones without quasigroup operations. We say that $C$ is constantive if it contains all unary constant operations.

Theorem 1.2. There exist a set $A$ with $|A|=\aleph_{0}$ and a constantive clone $C$ on $A$ such that $|\operatorname{Pol} \operatorname{Inv} C|=\aleph_{0}$ and $C \neq \operatorname{Pol} \operatorname{Inv} C$.

For a clone $C$ on $A, \operatorname{Inv}^{[m]} C$ denotes the set of $m$-ary invariant relations of $C$. It is well known that a function $f$ lies in Pol $\operatorname{Inv}^{[m]} C$ if and only if it can be interpolated at every $m$-element subset of its domain by a function in $C$; this is discussed, e.g., in [9] and in [4, Lemma 7] and stated in Lemma 3.1. We write $C^{[n]}$ for the set of $n$-ary functions in $C$. Let $B$ be any set, and let $F \subseteq A^{B}$. A subset $D$ of $B$ is a base of equality for $F$ if for all $f, g \in F$ with $\left.f\right|_{D}=\left.g\right|_{D}$, we have $f=g$. Theorem 1.1 can be extended in the following way:

Presented by R. Poeschel.

Received September 13, 2016; accepted in final form March 18, 2017.

2010 Mathematics Subject Classification: Primary: 08A40.

Key words and phrases: clones, local closure.

Supported by the Austrian Science Fund (FWF): P24077 and P29931. 
Theorem 1.3. Let $A$ be a set with $|A|=\aleph_{0}$, and let $C$ be a clone on $A$ with quasigroup operations. Then the following are equivalent:

(1) $\mid$ Pol Inv $C \mid \leq \aleph_{0}$.

(2) For each $n \in \mathbb{N}, C^{[n]}$ has a finite base of equality.

(3) $|C| \leq \aleph_{0}$ and $\forall n \in \mathbb{N} \exists k \in \mathbb{N}: C^{[n]}=\left(\text { Pol Inv }{ }^{[k]} C\right)^{[n]}$.

(4) $|C| \leq \aleph_{0}$ and $C=\operatorname{Pol} \operatorname{Inv} C$.

A weaker version of this result was proved in [1]. As an application, we obtain, e.g., that a countably infinite integral domain $R$ cannot be affine complete: If it is affine complete, then the clone $C$ of polynomial functions of $R$ satisfies (3), and therefore the unary polynomials have a finite base of equality $D$. But $f(x)=0$ and $g(x)=\prod_{d \in D}(x-d)$ show that this is not possible. In fact, Theorem 1.3 extracts a common idea of several "non-affine completeness" results $[6,8]$. The proofs are given in Section 4 .

\section{Finite bases of equality}

Theorems 1.1 and 1.3 rely on the following observation. In a less general context, this observation appears in [1, Theorem 2], and large parts of its proof are verbatim copies from [1] and [2, pp.51-52].

Lemma 2.1. Let $A$ be a set with $|A|=\aleph_{0}$, let $m \in \mathbb{N}$, and let $C$ be a clone on $A$ with quasigroup operations. If $\left|(\mathrm{Pol} \operatorname{Inv} C)^{[m]}\right| \leq \aleph_{0}$, then $C^{[m]}$ has a finite base of equality.

Proof. Let $\bar{C}:=\operatorname{Pol} \operatorname{Inv} C$. In the case that $\bar{C}^{[m]}$ is finite, its subset $C^{[m]}$ is also finite. Then for every $f, g \in C^{[m]}$ with $f \neq g$, we choose $a_{(f, g)} \in A^{m}$ such that $f\left(a_{(f, g)}\right) \neq g\left(a_{(f, g)}\right)$. Then $D:=\left\{a_{(f, g)} \mid f, g \in C^{[m]}, f \neq g\right\}$ is a base of equality for $C^{[m]}$. Hence, we will from now on assume $\left|\bar{C}^{[m]}\right|=\aleph_{0}$. Let $a_{0}, a_{1}, a_{2}, \ldots$ and $f_{0}, f_{1}, f_{2}, \ldots$ be complete enumerations of $A^{m}$ and $\bar{C}^{[m]}$, respectively. Furthermore, we abbreviate the set $\left\{a_{i} \mid i \leq r\right\}$ by $A(r)$. Seeking a contradiction, we suppose that there is no finite base of equality for $C^{[m]}$. We shall construct a sequence $\left(n_{k}\right)_{k \in \mathbb{N}_{0}}$ of non-negative integers and a sequence $\left(g_{k}\right)_{k \in \mathbb{N}_{0}}$ of elements of $C^{[m]}$ with the following properties:

(1) $\forall k \in \mathbb{N}_{0}:\left.g_{k}\right|_{A\left(n_{k}\right)} \neq\left. f_{k}\right|_{A\left(n_{k}\right)}$,

(2) $\forall k \in \mathbb{N}_{0}: n_{k+1}>n_{k}$,

(3) $\forall k \in \mathbb{N}_{0}:\left.g_{k+1}\right|_{A\left(n_{k}\right)}=\left.g_{k}\right|_{A\left(n_{k}\right)}$.

We construct the sequences inductively. We choose $g_{0} \in C^{[m]}$ such that $g_{0} \neq f_{0}$, and $n_{0} \in \mathbb{N}_{0}$ minimal with $g_{0}\left(a_{n_{0}}\right) \neq f_{0}\left(a_{n_{0}}\right)$. If we have already constructed $g_{k}$ and $n_{k}$, we construct $g_{k+1}$ and $n_{k+1}$ as follows: in the case that $\left.g_{k}\right|_{A\left(n_{k}\right)} \neq\left. f_{k+1}\right|_{A\left(n_{k}\right)}$, we set $g_{k+1}:=g_{k}$ and $n_{k+1}:=n_{k}+1$. In the case $\left.g_{k}\right|_{A\left(n_{k}\right)}=\left.f_{k+1}\right|_{A\left(n_{k}\right)}$, we first show that there exists a function $h \in C^{[m]}$ with

$$
\left.g_{k}\right|_{A\left(n_{k}\right)}=\left.h\right|_{A\left(n_{k}\right)} \text { and } h \neq f_{k+1} .
$$


Suppose that, on the contrary, every $h \in C^{[m]}$ with $\left.g_{k}\right|_{A\left(n_{k}\right)}=\left.h\right|_{A\left(n_{k}\right)}$ satisfies $h=f_{k+1}$. In this case, $g_{k}=f_{k+1}$, and therefore $f_{k+1} \in C^{[m]}$. We will show next that $A\left(n_{k}\right)$ is a base of equality of $C^{[m]}$. To this end, let $r, s \in C^{[m]}$ with $\left.r\right|_{A\left(n_{k}\right)}=\left.s\right|_{A\left(n_{k}\right)}$. We define $t(x):=r(x) \backslash\left(s(x) \cdot f_{k+1}(x)\right)$. Then for every $x \in A\left(n_{k}\right)$, we have $t(x)=r(x) \backslash\left(r(x) \cdot f_{k+1}(x)\right)=f_{k+1}(x)=g_{k}(x)$. Hence, $t=$ $f_{k+1}$. Therefore, for every $x \in A^{m}$, we have $r(x) \backslash\left(s(x) \cdot f_{k+1}(x)\right)=f_{k+1}(x)$, thus $s(x) \cdot f_{k+1}(x)=r(x) \cdot f_{k+1}(x)$, and therefore $\left(s(x) \cdot f_{k+1}(x)\right) / f_{k+1}(x)=$ $\left.\left(r(x) \cdot f_{k+1}(x)\right) / f_{k+1}(x)\right)$, which implies $s(x)=r(x)$. Thus, $r=s$, which completes the proof that $A\left(n_{k}\right)$ is a base of equality of $C^{[m]}$, contradicting the assumption that no such base exists. Hence, there is $h \in C^{[m]}$ that satisfies (2.1). Continuing in the construction of $g_{k+1}$, we set $g_{k+1}:=h$, and we choose $n_{k+1}$ to be minimal with $h\left(a_{n_{k+1}}\right) \neq f_{k+1}\left(a_{n_{k+1}}\right)$.

Since for every $a \in A^{m}$, the sequence $\left(g_{k}(a)\right)_{k \in \mathbb{N}_{0}}$ is eventually constant, we may define a function $l: A^{m} \rightarrow A$ by $l(a):=\lim _{k \rightarrow \infty} g_{k}(a)$. We will now show that $l \in \bar{C}^{[m]}$. The clone $\bar{C}$ contains exactly those functions that can be interpolated at every finite subset of their domain with a function in $C$. Hence, we show that $l$ can be interpolated at every finite subset $B$ of $A^{m}$ by a function in $C$. Since $\bigcup_{i \in \mathbb{N}_{0}} A_{i}=A^{m}$, there is $k \in \mathbb{N}$ such that $B \subseteq A\left(n_{k}\right)$. Since $\left.l\right|_{A\left(n_{k}\right)}=\left.g_{k}\right|_{A\left(n_{k}\right)}$, the function $g_{k} \in C^{[m]}$ interpolates $l$ at $B$. We conclude that the function $l$ lies in $\bar{C}^{[m]}$. Thus, $l$ is equal to $f_{k}$ for some $k \in \mathbb{N}_{0}$. Since $\left.l\right|_{A\left(n_{k}\right)}=\left.g_{k}\right|_{A\left(n_{k}\right)}$ and $\left.g_{k}\right|_{A\left(n_{k}\right)} \neq\left. f_{k}\right|_{A\left(n_{k}\right)}$, we obtain $\left.l\right|_{A\left(n_{k}\right)} \neq\left. f_{k}\right|_{A\left(n_{k}\right)}$, a contradiction. Hence, $C^{[m]}$ has a finite base of equality.

Lemma 2.2 (cf. [7, Lemma 1] and [1, Proposition 2]). Let $C$ be a clone on the set $A$, let $n \in \mathbb{N}$, let $D$ be a finite base of equality for $C^{[n]}$, and let $k:=|D|+1$. Then $C^{[n]}=\left(\operatorname{Pol} \operatorname{Inv}^{[k]} C\right)^{[n]}$.

Proof. Let $l \in\left(\mathrm{Pol} \mathrm{Inv}{ }^{[k]} C\right)^{[n]}$. Then $l$ can be interpolated at every subset of $A^{n}$ with at most $k$ elements by a function in $C^{[n]}$. Hence, there is $f \in C^{[n]}$ such that $\left.f\right|_{D}=\left.l\right|_{D}$. If $f=l$, then $l \in C^{[n]}$. In the case $f \neq l$, we take $y \in A^{n}$ such that $f(y) \neq l(y)$. Now we choose $g \in C^{[n]}$ such that $\left.g\right|_{D \cup\{y\}}=\left.l\right|_{D \cup\{y\}}$. Then $f(y) \neq g(y)$ and $\left.f\right|_{D}=\left.g\right|_{D}$, contradicting the assumption that $D$ is a base of equality for $C^{[n]}$.

\section{A compactness property for local interpolation}

For two sets $A$ and $B$, a set of functions $F \subseteq A^{B}$, and $k \in \mathbb{N}$, the set $\operatorname{Loc}_{k} F$ is defined as the set of those functions that can be interpolated at every subset of $B$ with at most $k$ elements by a function in $F$ [9]. If $C$ is a clone, and $F=C^{[m]}$ is its $m$-ary part, then $\operatorname{Loc}_{k}\left(C^{[m]}\right)$ is the set of $m$-ary functions on $A$ that preserve the $k$-ary relations in $\operatorname{Inv} C$.

Lemma 3.1 (cf. [9, p. 31, Theorem 4.1]). Let $C$ be a clone on the set $A$, and let $k, m \in \mathbb{N}$. Then $\operatorname{Loc}_{k}\left(C^{[m]}\right)=\left(\operatorname{Pol} \operatorname{Inv}{ }^{[k]} C\right)^{[m]}=\left(\operatorname{Pol~} \operatorname{Inv}^{[k]}\left(C^{[m]}\right)\right)^{[m]}$. 
For countable sets $A$, we obtain the following result.

Theorem 3.2. Let $A$ be a set with $|A| \leq \aleph_{0}$, and let $C$ be a clone on $A$ with quasigroup operations such that $\left|C^{[m]}\right| \leq \aleph_{0}$. If $\bigcap_{k \in \mathbb{N}} \operatorname{Loc}_{k}\left(C^{[m]}\right)=C^{[m]}$, then there exists $n \in \mathbb{N}$ such that $\operatorname{Loc}_{n}\left(C^{[m]}\right)=C^{[m]}$.

Proof. By Lemma 3.1 and the assumptions,

$$
C^{[m]}=\bigcap_{k \in \mathbb{N}} \operatorname{Loc}_{k}\left(C^{[m]}\right)=\bigcap_{k \in \mathbb{N}}\left(\operatorname{Pol} \operatorname{Inv}{ }^{[k]} C\right)^{[m]}=(\operatorname{Pol} \operatorname{Inv} C)^{[m]} .
$$

Now Lemma 2.1 yields a finite base of equality for $C^{[m]}$, and now by Lemma 2.2, there is $n \in \mathbb{N}$ such that $C^{[m]}=\left(\operatorname{Pol} \operatorname{Inv}{ }^{[n]} C\right)^{[m]}=\operatorname{Loc}_{n}\left(C^{[m]}\right)$.

For an arbitrary $m$-ary operation $f$ on the set $A$, we say that the property $I(f, n, C)$ holds if $f$ can be interpolated by a function in $C$ at each subset of $A^{m}$ with at most $n$ elements. Theorem 3.2 yields the following compactness property: if $C$ is a countable clone with quasigroup operations, if $A$ is countable, and if $\forall f \in A^{A^{m}}:((\forall k \in \mathbb{N}: I(f, k, C)) \Rightarrow f \in C)$ holds, then there is a natural number $n \in \mathbb{N}$ such that $\forall f \in A^{A^{m}}:(I(f, n, C) \Rightarrow f \in C)$ holds.

\section{Proofs of the theorems from Section 1}

Proof of Theorem 1.3. (1) $\Rightarrow(2)$ : Let $n \in \mathbb{N}$. Since $(\operatorname{Pol} \operatorname{Inv} C)^{[n]} \subseteq \operatorname{Pol} \operatorname{Inv} C$, we have $\mid(\operatorname{Pol} \text { Inv } C)^{[n]} \mid \leq \aleph_{0}$. Lemma 2.1 now yields a finite base of equality for $C^{[n]}$.

$(2) \Rightarrow(3)$ : Let $n \in \mathbb{N}$, and let $D \subseteq A^{n}$ be a finite base of equality for $C^{[n]}$. We set $k:=|D|+1$ and obtain $C^{[n]}=\left(\operatorname{Pol~Inv}{ }^{[k]} C\right)^{[n]}$ from Lemma 2.2. The mapping $\varphi: C^{[n]} \rightarrow A^{D},\left.f \mapsto f\right|_{D}$ is injective, therefore $\left|C^{[n]}\right| \leq \aleph_{0}$. Since for every $n \in \mathbb{N}$, we have $\left|C^{[n]}\right| \leq \aleph_{0}$, we have $|C| \leq \aleph_{0}$.

$(3) \Rightarrow(4)$ : Let $n \in \mathbb{N}$, and let $k$ be taken from (3). Then we have that $(\mathrm{Pol} \operatorname{Inv} C)^{[n]} \subseteq\left(\mathrm{Pol} \mathrm{Inv}{ }^{[k]} C\right)^{[n]}=C^{[n]}$.

$(4) \Rightarrow(1)$ : This is obvious.

Proof of Theorem 1.1. The statement of Theorem 1.1 is given by the implication $(1) \Rightarrow(4)$ of Theorem 1.3.

Proof of Theorem 1.2. Let $A:=\mathbb{N}_{0}$, and let $p(x):=x \bmod 2$ for all $x \in \mathbb{N}_{0}$. For $a \in \mathbb{N}_{0}$, we define $g_{a}: \mathbb{N}_{0} \rightarrow \mathbb{N}_{0}$ by

$$
g_{a}(x):= \begin{cases}p(x) & \text { if } x<a, \\ x & \text { if } x \geq a,\end{cases}
$$

with $c_{a}(x):=a$ for all $x \in \mathbb{N}_{0}$. Let $M:=\left\{g_{a} \mid a \in \mathbb{N}_{0}\right\} \cup\left\{c_{a}(x) \mid a \in \mathbb{N}_{0}\right\}$. We will first show that $\left\langle M, \circ, g_{0}\right\rangle$ is a submonoid of $\left\langle\mathbb{N}_{0}{ }^{\mathbb{N}_{0}}, \circ, \mathrm{id}_{\mathbb{N}_{0}}\right\rangle$. To this end, it is sufficient to show that $g_{a} \circ g_{b} \in M$ for all $a, b \in \mathbb{N}_{0}$. Since $g_{0}=g_{1}=g_{2}=\mathrm{id}_{\mathbb{N}_{0}}$, we may assume $a \geq 3$ and $b \geq 3$. We will show

$$
g_{a}\left(g_{b}(x)\right)=g_{\max (a, b)}(x) \text { for all } x \in \mathbb{N}_{0} .
$$


If $x<b$, then $g_{a}\left(g_{b}(x)\right)=g_{a}(p(x))=p(p(x))=p(x)=g_{\max (a, b)}(x)$. If $x \geq b$ and $x<a$, then $g_{a}\left(g_{b}(x)\right)=g_{a}(x)$; since in this case $b \leq a$, so $g_{a}(x)=$ $g_{\max (a, b)}(x)$. If $x \geq a$ and $x \geq b$, then $g_{a}\left(g_{b}(x)\right)=g_{a}(x)=x=g_{\max (a, b)}(x)$. From (4.1), we deduce that $M$ is closed under composition. Now let $C$ be the clone on $\mathbb{N}_{0}$ that is generated by $M$; this clone consists of all functions $\left(x_{1}, \ldots, x_{n}\right) \mapsto m\left(x_{j}\right)$ with $n, j \in \mathbb{N}, m \in M$ and $j \leq n$. Let $\bar{C}:=\operatorname{Pol} \operatorname{Inv} C$. Next, we show

$$
p \in \bar{C} .
$$

To prove (4.2), we show that $p$ can be interpolated at every finite subset $B$ of $\mathbb{N}_{0}$ by a function in $C$. Let $a:=\max (B)$. Then $\left.g_{a+1}\right|_{B}=\left.p\right|_{B}$. This completes the proof of (4.2). Now we show

$$
\bar{C}^{[1]}=C^{[1]} \cup\{p\} .
$$

We only have to establish $\subseteq$. It is helpful to write down the list of values of some of the functions in $M \cup\{p\}$.

$\begin{array}{rl}c_{3} & 333333 \ldots \\ c_{2} & 222222 \ldots \\ c_{1} & 111111 \ldots \\ c_{0} & 000000 \ldots \\ p & 010101 \ldots \\ \mathrm{id} & 012345 \ldots \\ g_{3} & 010345 \ldots \\ g_{4} & 010145 \ldots \\ g_{5} & 010105 \ldots\end{array}$

Let $f \in \bar{C}^{[1]}$ with $f \neq p$, and let $k \in \mathbb{N}_{0}$ be minimal with $f(k) \neq p(k)$. Let $g \in C^{[1]}$ be such that $\left.g\right|_{\{0, \ldots, k\}}=\left.f\right|_{\{0, \ldots, k\}}$. We distinguish three cases.

Case $k=0$ : Then $g(0) \neq 0$, and therefore $g=c_{g(0)}$. If $f=c_{g(0)}$, we have $f \in C$. If $f \neq c_{g(0)}$, we let $y$ be minimal with $f(y) \neq g(0)$. We interpolate $f$ at $\{0, y\}$ by a function $h \in C$. This function $h$ is not constant and satisfies $h(0) \neq 0$. Such a function does not exist in $C$, therefore the case $f \neq c_{g(0)}$ cannot occur.

Case $k=1$ : Then $g(1) \neq 1$. By examining the functions in $M$, we see that $g=c_{0}$. If $f=c_{0}$, we have $f \in C$. If $f \neq c_{0}$, we let $y$ be minimal with $f(y) \neq 0$. Interpolating $f$ at $\{0,1, y\}$ by $h \in C$, we obtain a function $h \in C$ with $h(0)=h(1)=0$ and $h(y) \neq 0$. Such a function does not exist in $C$; this contradiction shows $f=c_{0}$ and therefore $f \in C$.

Case $k \geq 2$ : Then $g=g_{k}$. If $f=g_{k}$, then $f \in C$. If $f \neq g_{k}$, we choose $y$ minimal with $f(y) \neq g_{k}(y)$ and interpolate $f$ at $\{0,1, \ldots, k\} \cup\{y\}$ by a function $h \in C$. Again, such a function is not available in $C$, and therefore $f=g_{k} \in C$.

Thus, every $f \in \bar{C}^{[1]}$ with $f \neq p$ is an element of $C$. By its definition, $C$ contains all constant unary operations in $\mathbb{N}_{0}$. Since $C$ preserves the relation 
$\rho=\left\{(a, b, c, d) \in A^{4} \mid a=b\right.$ or $\left.c=d\right\}, \bar{C}$ also preserves $\rho$. Therefore, by [10, Lemma 1.3.1(a)], every function in $\bar{C}$ is essentially unary and hence of the form $l\left(x_{1}, \ldots, x_{n}\right)=f\left(x_{j}\right)$ with $n \in \mathbb{N}, j \in\{1, \ldots, n\}$, and $f \in \bar{C}^{[1]}=M \cup\{p\}$. This implies that $\bar{C}$ is countable. The function $p$ witnesses $C \neq \bar{C}$.

\section{Constantive clones}

In constantive clones, a finite base of equality for the functions of arity $m$ yields finite bases of equality for all other arities. This will allow us to refine Theorem 1.3.

Lemma 5.1. Let $C$ be a clone on the set $A$, let $m \in \mathbb{N}$, and let $D \subseteq A^{m}$ be a base of equality for $C^{[m]}$. Then the projection of $D$ to the first component $\pi_{1}(D)$ is a base of equality for $C^{[1]}$.

Proof. Let $f, g \in C^{[1]}$ with $\left.f\right|_{\pi_{1}(D)}=\left.g\right|_{\pi_{1}(D)}$. Let $f_{1}\left(x_{1}, \ldots, x_{m}\right):=f\left(x_{1}\right)$ and $g_{1}\left(x_{1}, \ldots, x_{m}\right):=g\left(x_{1}\right)$. Then for every $\left(d_{1}, \ldots, d_{m}\right) \in D$, we have $f_{1}\left(d_{1}, \ldots, d_{m}\right)=f\left(d_{1}\right)=g\left(d_{1}\right)=g_{1}\left(d_{1}, \ldots, d_{m}\right)$, and therefore $f_{1}=g_{1}$, which implies $f=g$.

Lemma 5.2. Let $A$ be a set, let $C$ be a constantive clone on $A$, and let $D \subseteq A$ be a base of equality for $C^{[1]}$. Then for every $n \in \mathbb{N}, D^{n}$ is a base of equality for $C^{[n]}$.

Proof. We proceed by induction on $n$. If $n=1, D^{1}=D$ is a base of equality of $C^{[1]}$ by assumption. For the induction step, let $n \geq 2$, and suppose that $D^{n-1}$ is a base of equality for $C^{[n-1]}$. Let $f, g \in C^{[n]}$ and assume $\left.f\right|_{D^{n}}=\left.g\right|_{D^{n}}$. We first show

$$
\left.f\right|_{A \times D^{n-1}}=\left.g\right|_{A \times D^{n-1}} .
$$

Let $\left(a, d_{2}, \ldots, d_{n}\right) \in A \times D^{n-1}$, and define $f_{1}(x):=f\left(x, d_{2}, \ldots, d_{n}\right)$ and $g_{1}(x):=g\left(x, d_{2}, \ldots, d_{n}\right)$ for $x \in A$. Then $f_{1}, g_{1} \in C^{[1]}$ and $\left.f_{1}\right|_{D}=\left.g_{1}\right|_{D}$. Hence, $f_{1}=g_{1}$, and thus $f\left(a, d_{2}, \ldots, d_{n}\right)=f_{1}(a)=g_{1}(a)=g\left(a, d_{2}, \ldots, d_{n}\right)$, which completes the proof of (5.1).

We prove $f=g$. Let $\left(b_{1}, \ldots, b_{n}\right) \in A^{n}$; for all $x_{2}, \ldots, x_{n} \in A$, define $f_{2}\left(x_{2}, \ldots, x_{n}\right):=f\left(b_{1}, x_{2}, \ldots, x_{n}\right)$ and $g_{2}\left(x_{2}, \ldots, x_{n}\right):=g\left(b_{1}, x_{2}, \ldots, x_{n}\right)$. By (5.1), $\left.f_{2}\right|_{D^{n-1}}=\left.g_{2}\right|_{D^{n-1}}$, and thus by the induction hypothesis, $f_{2}=g_{2}$. Thus, $f\left(b_{1}, \ldots, b_{n}\right)=g\left(b_{1}, \ldots, b_{n}\right)$.

Hence, for constantive clones we can give the following slight refinement of Theorem 1.3.

Theorem 5.3. Let $A$ be a set with $|A|=\aleph_{0}$, let $C$ be a constantive clone on $A$ with quasigroup operations, and let $m \in \mathbb{N}$. Then the following are equivalent:

(1) $\left|(\operatorname{Pol} \operatorname{Inv} C)^{[1]}\right| \leq \aleph_{0}$.

(2) $C^{[1]}$ has a finite base of equality.

(3) $C^{[m]}$ has a finite base of equality. 
(4) $|C| \leq \aleph_{0}$ and $\exists d \in \mathbb{N} \forall n \in \mathbb{N}: C^{[n]}=\left(\operatorname{Pol~Inv}{ }^{\left[d^{n}+1\right]} C\right)^{[n]}$.

(5) $|C| \leq \aleph_{0}$ and $\forall n \in \mathbb{N} \exists k \in \mathbb{N}: C^{[n]}=\left(\mathrm{Pol} \mathrm{Inv}^{[k]} C\right)^{[n]}$.

(6) $|C| \leq \aleph_{0}$ and $C=\operatorname{Pol} \operatorname{Inv} C$.

Proof. $(1) \Rightarrow(2)$ : This is Lemma 2.1 .

$(2) \Rightarrow(3)$ : This is Lemma 5.2 .

$(3) \Rightarrow(2)$ : This is Lemma 5.1 .

$(2) \Rightarrow(4)$ : Let $D$ be a finite base of equality for $C^{[1]}$. Let $n \in \mathbb{N}$, and set $k:=|D|^{n}+1$. By Lemma $5.2, D^{n}$ is a base of equality for $C^{[n]}$, and Lemma 2.2 yields $C^{[n]}=\left(\mathrm{Pol} \mathrm{Inv}{ }^{[k]} C\right)^{[n]}$. Since $D^{n}$ is a finite base of equality, the mapping $\left.f \mapsto f\right|_{D^{n}}$ is an injective mapping from $C^{[n]}$ to $A^{D^{n}}$, making $C^{[n]}$ countable. Since $C^{[n]}$ is countable for every $n \in \mathbb{N}$, we obtain $|C| \leq \aleph_{0}$.

$(4) \Rightarrow(5):$ Set $k:=d^{n}+1$.

$(5) \Rightarrow(6)$ : Let $n \in \mathbb{N}$, and let $k$ be produced by (5). Then we have that $(\mathrm{Pol} \text { Inv } C)^{[n]} \subseteq\left(\mathrm{Pol} \mathrm{Inv}{ }^{[k]} C\right)^{[n]}=C^{[n]}$.

$(6) \Rightarrow(1)$ : We have $(\text { Pol Inv } C)^{[1]} \subseteq$ Pol Inv $C \subseteq C$.

Acknowledgements. Open access funding provided by Johannes Kepler University Linz. The author thanks Mike Behrisch for furnishing information on the reference [9].

\section{REFERENCES}

[1] Aichinger, E.: Local polynomial functions on the integers. Riv. Mat. Univ. Parma (5) 6, 169-177 (1997)

[2] Aichinger, E.: The structure of composition algebras. Ph.D. thesis, Johannes Kepler Universität Linz (1998). Available at www.algebra.uni-linz.ac.at/ erhard/Diss/

[3] Burris, S., Sankappanavar, H.P.: A course in universal algebra. Springer New York Heidelberg Berlin (1981)

[4] Eigenthaler, G.: Einige Bemerkungen über Clones und interpolierbare Funktionen auf universellen Algebren. Beiträge Algebra Geom. 15, 121-127 (1983)

[5] Goldstern, M., Pinsker, M.: A survey of clones on infinite sets. Algebra Universalis 59, 365-403 (2008)

[6] Hall, R.R.: On pseudo-polynomials. Mathematika 18, 71-77 (1971)

[7] Hule, H., Nöbauer, W.: Local polynomial functions on universal algebras. Anais da Acad. Brasiliana de Ciencias 49, 365-372 (1977)

[8] Kaarli, K.: Affine complete abelian groups. Math.Nachr. 107, 235-239 (1982)

[9] Pöschel, R.: A general Galois theory for operations and relations and concrete characterization of related algebraic structures, Report 1980, vol. 1. Akademie der Wissenschaften der DDR, Institut für Mathematik, Berlin (1980)

[10] Pöschel, R., Kalužnin, L.A.: Funktionen- und Relationenalgebren, Mathematische Monographien, vol. 15. VEB Deutscher Verlag der Wissenschaften, Berlin (1979)

[11] Szendrei, Á.: Clones in universal algebra. Séminaire de Mathématiques Supérieures [Seminar on Higher Mathematics], vol. 99. Presses de l'Université de Montréal, Montreal, QC (1986)

\section{ERHARD Aichinger}

Institute for Algebra, Johannes Kepler University Linz, 4040 Linz, Austria e-mail: erhard@algebra.uni-linz.ac.at

$U R L:$ http://www.jku.at/algebra

Open Access This article is distributed under the terms of the Creative Commons Attribution 4.0 International License (http://creativecommons.org/licenses/by/4.0/), which permits unrestricted use, distribution, and reproduction in any medium, provided you give appropriate credit to the original author(s) and the source, provide a link to the Creative Commons license, and indicate if changes were made. 